\title{
The Ras Dimer Structure
}

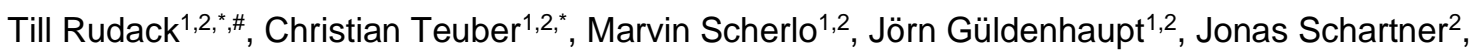
Mathias Lübben ${ }^{1,2}$, Johann Klare ${ }^{3}$, Klaus Gerwert ${ }^{1,2, \#}$, Carsten Kötting ${ }^{1,2, \#}$

${ }^{1}$ Biospectroscopy, Center for Protein Diagnostics, Ruhr University Bochum, 44801 Bochum, Germany

${ }^{2}$ Department of Biophysics, Ruhr University Bochum, 44801 Bochum, Germany

${ }^{3}$ Department of Physics, Osnabrück University, 49074 Osnabrück, Germany

* These authors contributed equally to this work

\# Corresponding author

Till.Rudack@ruhr-uni-bochum.de

Klaus.Gerwert@ruhr-uni-bochum.de

Carsten.Koetting@ruhr-uni-bochum.de

Keywords: GTPase, cancer, drug target, unnatural amino acids, FRET, EPR, Integrative Modeling 


\begin{abstract}
Oncogenic mutated Ras is a key player in cancer, but despite intense and expensive approaches its catalytic center seems undruggable. The Ras dimer interface is a possible alternative drug target. Dimerization at the membrane affects cell growth signal transduction. In vivo studies indicate that preventing dimerization of oncogenic mutated Ras inhibits uncontrolled cell growth. Conventional computational drug-screening approaches require a precise atomic dimer model as input to successfully access drug candidates. However, the proposed dimer structural models are controversial. Here, we provide a clear-cut experimentally validated N-Ras dimer structural model. We incorporated unnatural amino acids into Ras to enable the binding of labels at multiple positions via click chemistry. This labeling allowed for the determination of multiple distances of the membrane-bound Ras-dimer measured by fluorescence and electron paramagnetic resonance spectroscopy. In combination with protein-protein docking and biomolecular simulations we identified key residues for dimerization. Site-directed mutations of these residues prevent dimer formation in our experiments, proving our dimer model to be correct. The presented dimer structure enables now computational drug-screening studies exploiting the Ras dimer interface as alternative drug target.
\end{abstract}




\section{Introduction}

Ras is a small G Protein that acts as a molecular switch in the context of cell differentiation, proliferation, and growth. Ras is active in the guanosine triphosphate (GTP) bound state and transduces signals downstream to the cell nucleus. Furthermore, it catalyzes the hydrolysis from GTP to guanosine diphosphate (GDP) leading to an inactive state. This switch-off process is further accelerated by GTPase-activating proteins (GAPs) ${ }^{1}$. If the catalysis function of Ras is impaired by mutations, tumors can develop due its constitutively active state. The reaction mechanisms of the GTP hydrolysis has been well characterized using experimental techniques such as X-Ray structure analysis, ${ }^{2,}{ }^{3}$ nuclear magnetic resonance spectroscopy, ${ }^{4}$ Fourier transform infrared (FTIR) spectroscopy, ${ }^{5}$ and theoretical methods ${ }^{6}$. Both, the allosteric regulation of Ras and its signaling ${ }^{7-10}$ as well as insights into catalysis of small GTPases have been previously reviewed. ${ }^{1,11,10,12,13}$

Currently, there is no drug against constitutively active Ras approved and Ras is traditionally referred to as undruggable. Recently, with advancing methods and new targeting concepts, progress was made in Ras targeting drugs development. ${ }^{14}$ This progress has led to the first drug; however its effects are restricted to a relatively uncommon mutation $(\mathrm{G} 12 \mathrm{C})^{15}{ }^{16}$. In this case a covalent attachment to the $\mathrm{C} 12$ allows the interference with the otherwise hard to target catalytic center.

Because targeting the catalytic center is so difficult many efforts are being made to study Ras in a larger context, including its processing, interaction partners, distribution, and organization at the plasma membrane ${ }^{10}$. Ras dimerization and its impact on Raf- 1 activation was described first in the year $2000 .{ }^{17}$ In 2013 the first in vitro structural model of a Ras dimer at a membrane was proposed based on attenuated total reflectance infrared spectroscopy measurements in combination with distances estimated by Förster resonance energy transfer (FRET) using fluorescently labeled nucleotides and biomolecular simulations. ${ }^{18}$ This study initiated several follow up studies that showed dimerization in vivo ${ }^{19-21}$ opening up a new strategy for cell growth signaling inhibition by disrupting the proposed dimerization interface. The enhanced dimerization was shown to activate downstream signaling ${ }^{20}$ while inhibition of dimer formation by a small protein ${ }^{19}$ led to signal interference. In vitro and in vivo the presence of K-Ras dimers was demonstrated by GFP based FRET measurements. ${ }^{22}$ The same study showed that a dimer preventing mutation of oncogenic K-Ras (K-Ras $\left.{ }^{\mathrm{G} 12 \mathrm{D} / \mathrm{D} 154 \mathrm{Q}}\right)$ inhibits tumor growth in mice ${ }^{22}$. Using quantitative photoactivated localization microscopy it was shown that K-Ras-GDP and K-RasGTP form dimers at a physiological expression level. ${ }^{20}$ Dynamic light scattering experiments showed K-Ras4B dimer formation with the bound nucleotide analogue GTPYS. ${ }^{23}$ Nevertheless, there have been conflicting reports about Ras dimerization. ${ }^{10,24}$

Kovrigina et al. analyzed the time-domain fluorescence anisotropy and NMR chemical shift perturbations of the Ras G-domain without a lipid anchor and they conclude that the Ras Gdomain alone does not form dimers in solution. ${ }^{25}$ In addition, Groves and colleagues showed in vitro that full-length and fully processed K-Ras4B remains monomeric in supported lipid bilayer membranes under variable conditions. ${ }^{26}$ It was shown that photosensitized oxidation, due to experimental conditions such as high laser intensities, may lead to Ras dimerization. ${ }^{27}$ Furthermore, high protein concentration might lead to artificial dimerization as well.

However, the majority of studies support the presence of membrane bound Ras dimers. Immune electron microscopy studies show that about $40 \%$ of the Ras proteins form nanoclusters with a radius of approximately $9 \mathrm{~nm}$ at the membrane. ${ }^{28,29}$ In one case even a trimeric organization of K-Ras has been observed, ${ }^{30}$ while the rest of the observed organizations showed dimerization 
independent of the isoform. ${ }^{18,23,31,32}$ The Ras clusters act as isoform specific signaling platforms that recruit and activate effectors. ${ }^{33-35}$ For targeted development of anti-cancer drugs that break dimers of malfunctioning Ras and thereby inhibit signaling, a detailed understanding of the exact dimer interaction network is desirable. ${ }^{36}$

Promising drug targets can be proposed based on atomic level information regarding dimer interactions. However, the exact molecular structure of Ras-dimers remains elusive. Figure 1 illustrates the diversity of the reported ${ }^{18,23,31,32}$ various different contradictory dimer structural models. We categorize these models into three main categories based on their dimer interaction sides. The models in category I undergo helix $\alpha 4$ and a5 dimerization, the ones in category II undergo helix $\alpha 3$ and $\alpha 4$ dimerization, while the ones in category III undergo $\beta$-sheet dimerization. These categories are further subdivided with respect to their detailed dimer interactions in Fig. S1. Structure I.1 was derived from the crystal contacts within most Ras X-ray structures, $^{18}$ I.2 (either N-Ras-GDP ${ }^{18}$ or K-Ras4B-GTP ${ }^{31}$ ) was obtained by biomolecular simulations, I.3 (K-Ras4B-GDP) and I.4 (K-Ras4B-GTP) were both derived from NMR data. ${ }^{32}$ Structure II.1 (K-Ras4B-GTP) was obtained by biomolecular simulations ${ }^{31}$, while II.2 (K-Ras4BGppNHp) and III.1 (K-Ras4B-GTP- $\gamma-S)^{23}$ were generated by the protein interactions by structural matching (PRISM) algorithm ${ }^{37}$. Details about the models are given in supporting note 1.
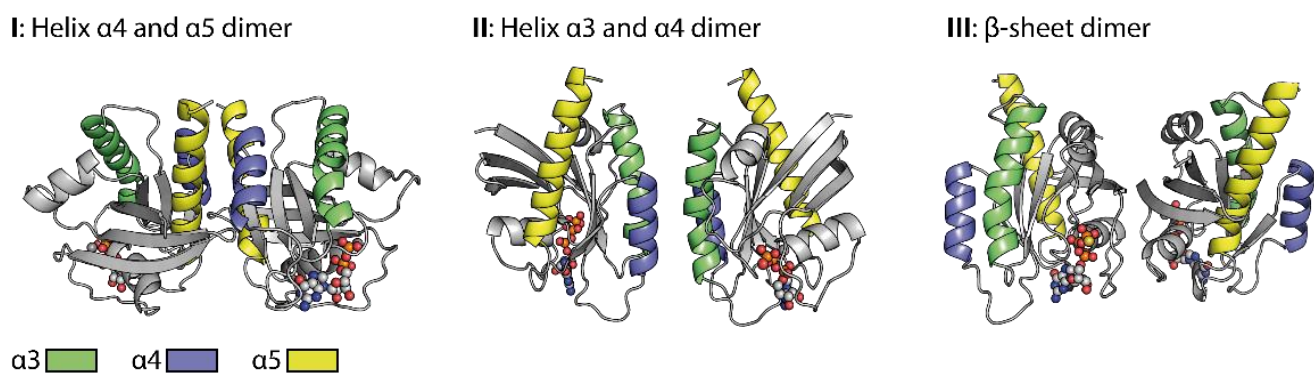

Fig. 1. Diversity of proposed Ras dimer structural models. To order the huge variety of the published dimer structural models we divided them into three substantially different main categories based on the relative orientation of the monomers. The key helices are represented by different colors (helix a3: green, helix a4: blue, helix a5: yellow) and the nucleotides are displayed in spherical shape. The category I dimer interface is formed by contacts of helices $\alpha 4$ and $\alpha 5$, category II by helix $\alpha 3$ and $\alpha 4$ contacts, and category III by $\beta$-sheet contacts.

While various aforementioned experimental data indicate Ras dimerization, only few studies provide insights into the structure of such dimers at the molecular level with atomic resolution. Two studies obtained NMR shifts for K-Ras4B dimers, one without membrane ${ }^{23}$ and one using nanodiscs to mimic the membrane attachment ${ }^{32}$. However, the structural models reported, namely model I.3, I.4, II.2, and III.1, vary over all three categories. In a previous study we measured a FRET distance of $46 \pm 6 \AA$ between the labeled (Mant/TNP) nucleotides. ${ }^{18}$ The aim of this study was to identify Ras dimerization and its orientation attachment to the membrane. However, the distance between labeled nucleotides measured with FRET is not sufficient to unambiguously determine the correct orientation of the monomers within a dimer. Overall, while there are various different dimer structural models reported, none of them are clear-cut experimentally validated. Therefore, additional experimental data are required to allow for an unambiguous validation of the proposed dimer structural models.

In order to obtain dimer structural models, we first used different established docking algorithms to predict dimer structural models with satisfactory statistical accuracy. Next, we employed FRET and electron paramagnetic resonance (EPR) spectroscopy to provide several experimental 
distances to enable an unambiguous validation for the predicted models. For these methods, fluorophores or spin labels need to be attached to various positions of the Ras surface to obtain sufficient distance variations for each structural model. To achieve membrane binding, a lipid anchor is attached to the Ras protein via maleimide chemistry at Cys181 limiting the chemical options to introduce labels. Therefore, to ensure flexible site specific labeling for the fluorophores and the EPR label we developed a strategy to incorporate unnatural amino acids into Ras. These amino acids were used as binding sites to which fluorophores or spin labels were attached via a second biorthogonal click reaction that does not cross-interfere with the lipid coupling via the maleimide group. The identified dimer structural models that coincided with the available experimental FRET and EPR distances were further refined by biomolecular simulations to equilibrate the solvated membrane attached models. Based on these simulations we identified key dimer forming interaction residues. The effects of mutations of the computationally suggested interaction on dimerization were further checked experimentally by site-specific mutagenesis and FRET. A reliable characterization of the atomic details of Ras dimer interfaces is needed to guide the search for drug target sites for small molecules, which exert their anti-cancer effects by impairing Ras dimerization and constitutive signaling.

\section{Results}

\section{Computational prediction of possible Ras dimer structural models}

The literature shows that different workflows and docking algorithms provide numerous different Ras dimer models. We divide the seven published highly divers structural models into three main categories (I-III) based on their contact sides (Fig. 1). In order to obtain more potential dimer structures we used three different protein-protein docking web servers $\left(\mathrm{PRISM}^{37}, \mathrm{ZDOCK}^{38}\right.$ and Symmdock $\left.{ }^{39},{ }^{40}\right)$. As input, we used six monomeric structural models of the Ras G-domain (residues 1 to 172) for H-Ras, K-Ras4B, and N-Ras with bound GTP and GDP. Table S1 summarizes the resulting 178 different dimer structural models reflecting nine different main interaction sides. While three of these interaction sides were already identified in the published models, six new possible interaction sides were detected by these algorithms (IV-IX). Details are given in supporting note 2 .

\section{Experimental strategy to obtain different experimental distances}

To reduce the number of possible dimer models and enable a clear-cut assignment of a unique structural dimer model, we developed a new strategy (Fig. 2) to obtain additional experimental distance information. The number of natural binding sites for labels to measure distances is limited and site specificity is difficult to achieve. Thus, the N-Ras lipid anchor containing a palmitoyl and a farnesyl group, was attached to cysteine 181 of $\mathrm{N}$-Ras via a maleimide group (See Fig. 2A and supporting note 3 ). The chemistry necessary to attach the anchor limits the chemical options to introduce labels for distance measurements. Individual labeling positions for FRET and EPR measurements were achieved by incorporation of unnatural amino acids. The used strategy is described in Fig. S2, and is based on previously reported protocols from Edward Lemke $^{41}$, which describe the site specific incorporation of the unnatural amino acids N-propargylL-lysine (PrK) and the cyclooctyne-lysine (SCO) into N-Ras using the amber codon suppression strategy. The unnatural amino acids bind the fluorophores or spin labels without interfering with lipid anchor binding. We identified S106 and T124 as the best incorporation sites yielding sufficient amounts of protein with the incorporated unnatural amino acid (Fig. S2B). 


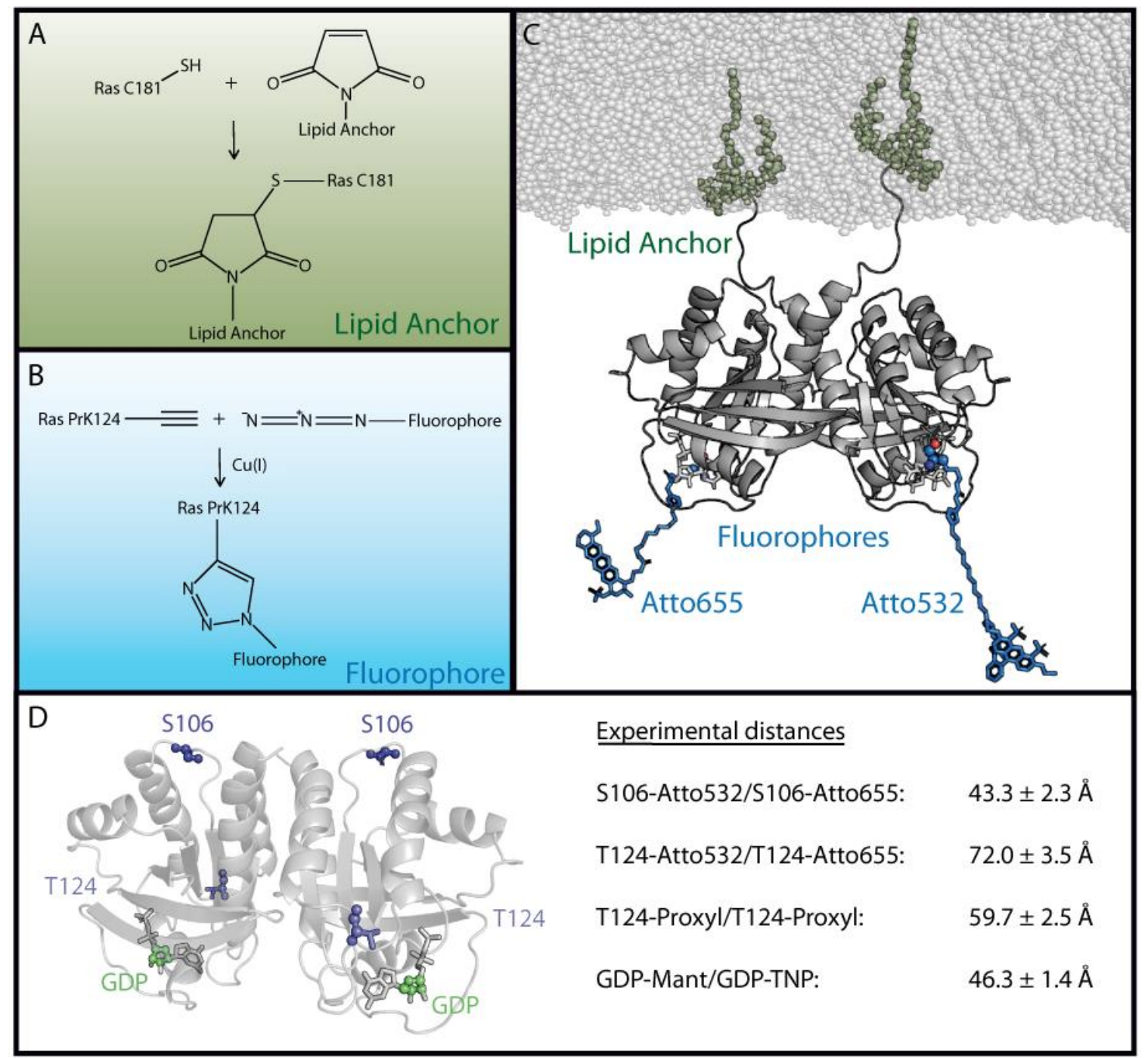

Fig. 2. Experimental strategy for the attachment of lipid anchor and fluorophores to enable FRET or EPR measurements of membrane bound Ras proteins. In order to perform FRET or EPR measurements on membrane-bound Ras, two site specific protein modifications were necessary; one to attach the lipid anchor to the protein and another for the fluorophore or spin label. (A) The lipid anchor is attached via a maleimide group to ensure membrane binding. (B) The fluorophore is coupled site specifically via copper (I) catalyzed alkyne-azide cycloaddition to the previously incorporated unnatural amino acid (Fig. S2). (C) A Ras dimer structural model with attached lipid anchors and the fluorophore pair T124-Atto 655/T124-Atto 532. (D) An overview of the labeling sites and the experimentally obtained FRET and EPR distances between two membrane bound full-length N-Ras-GDP proteins. The previously reported GDP distance was used $^{18}$. 
Next, the fluorophores and spin labels are bound to the unnatural amino acids via the copper (I) catalyzed alkyne- azide cycloaddition (CuAAC) as shown in Fig. 2B and supporting note 3 or via the strain promoted alkyne-azide cycloaddition (SPAAC). One fraction of PrK labelled Ras was bound to Atto 532 azide (FRET donor) and another fraction was bound to Atto 655 azide (FRET acceptor). Similarly, the EPR spin label azido-proxyl was coupled to T124 of N-Ras via the unnatural amino acid SCO. Figure 2C shows exemplarily a Ras dimer structural model with attached lipid anchor and the fluorophores Atto 655 and Atto 532 at position T124.

Then, we experimentally determined the FRET distances summarized in Fig. 2D. These distances are based on FRET efficiencies, which were derived from the fluorophore lifetimes measured with a time-correlated single photon counting (TCSPC) setup. The respective TCSPC histograms of all measured FRET distances and the experimental EPR data are presented in Fig. S3. For these FRET measurements, we mixed the Ras protein to be investigated with 1-palmitoyl-2-oleoyl-sn-phosphatidylcholine (POPC) liposomes. The lifetime (T) for membranebound N-Ras (GDP) S106-Atto 532 was analyzed in the presence (TDA) and absence (TD) of the acceptor N-Ras (GDP) S106-Atto 655 (Fig. S3A). The analog experiment was performed for membrane-bound N-Ras (GDP) T124-Atto532 and the acceptor N-Ras (GDP) T124-Atto 655 (Fig. S3B). Both resulting histograms show a reduced TDA compared to TD due to FRET. The T reduction is significantly stronger in samples with N-Ras (GDP) S106-Atto 532 (from 1.87 ns to $1.28 \mathrm{~ns}$ ) than in samples with N-Ras (GDP) T124-Atto 532 (from 2.66 ns to $2.53 \mathrm{~ns}$ ). Due to the large distance between the fluorophores at the T124 position, we verified the T124-T124 distance independently with an in this distance regime more accurate EPR spectroscopic double electronelectron resonance (DEER) measurement method (Fig. S3C). This experiment resulted in a time-dependent echo amplitude that has been described by a gaussian fit in order to obtain the distance information. The distance between the nucleotides was previously determined by FRET (18) (Fig. S3D). The experimental details of the distance determination process are summarized in the methods section, supporting note 4 , and in Table S2.

The obtained distances between two membrane-bound lipidated N-Ras monomers (Fig. 2D) are $43.3 \pm 2.3 \AA$ between the FRET fluorophores coupled to S106-S106, $72 \pm 3.5 \AA$ between the fluorophores coupled to T124-T124, $59.7 \pm 2.5 \AA$ between the EPR proxyl labels at T124-T124, and $46.3 \pm 1.4 \AA$ between the fluorescent labeled nucleotides ${ }^{18}$. The distance between the EPR labels at T124 is shorter than the one between the FRET labels at the same position, as the EPR label is shorter than the FRET label (Fig. S4). The corresponding Ca distance is the same for both methods. The three different labeling positions are well distributed over the Ras surface as shown in Fig. 2D, which ensures a significantly more precise validation of possible Ras dimer structural models than the previously available single distance of the labeled nucleotides.

The obtained distances for two N-Ras monomers including the lipid anchor solely in solution without membrane are $49.7 \pm 0.8 \AA$ between the FRET fluorophores coupled to S106-S106 and $77 \pm 0.5 \AA$ between the ones coupled to T124-T124. All distances are summarized in Table S2.

\section{Comparison of experimental distances with calculated distances of the predicted dimer models}

We utilized the four experimental distances for membrane-bound N-Ras-GDP to evaluate the 178 dimer conformations resulting from our protein-protein docking (Table S1). The nine main conformations of the monomer orientation are further divided into 64 subcategories, representing slight variations in the dimer binding interface. Next, we calculated the respective distance distributions for each of the 64 conformations within the subcategories and compared them with the experimentally obtained values. Details regarding the generation of rotamer ensembles and 
calculation of the respective distance distributions for all experimentally used labels and positions are shown in Fig. S4 and supporting note 5. We used the PyMOL plugin MTSSL Wizard to calculate the rotamer ensembles. The distributions are fitted by a gaussian function to obtain a mean value and standard deviation for each labeling position of each dimer conformation (Table S3). As a further control of the label ensembles created by the MTSSL Wizard, we performed biomolecular simulations to analyze the conformational space sampled by SCO-Proxyl at T124 (Fig. S5).
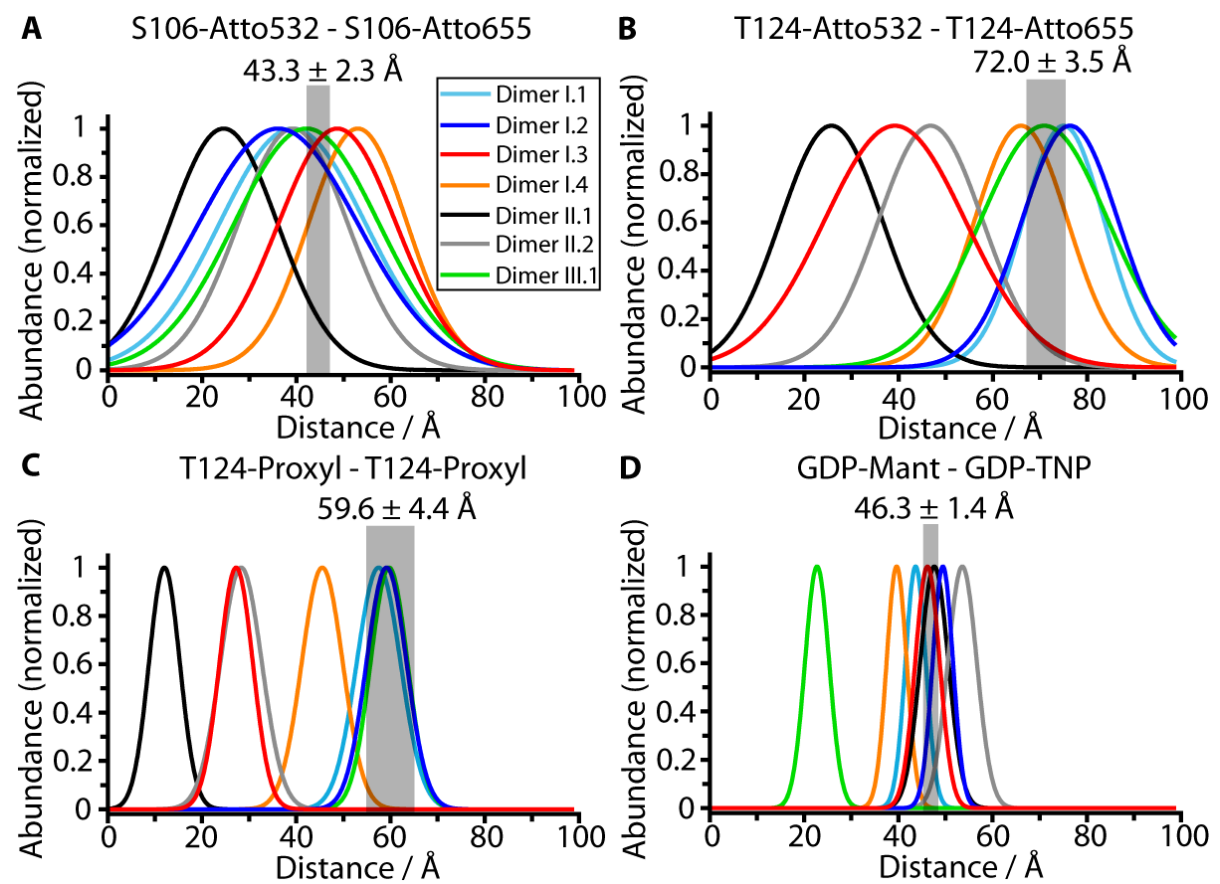

Fig. 3. Comparison of the experimental distances with the calculated distances for predicted dimer structural models. The experimental values obtained by FRET and EPR measurements (Fig. S3) are shown as transparent grey bars. Only dimer structural models I.1 and 1.2 are in line with all four measured distances as revealed by the comparison of the measured distances S106-S106 (A), T124-T124 (B), T124-T124 (EPR) (C) and nucleotidenucleotide (D) (grey bars) with the calculated distance distributions based on the dimer structural models I.1 (light blue), I.2 (dark blue), I.3 (cyan), II.1 (black), II.2 (grey) and III.1 (green) as detailed in supporting note 5 . The width of the calculated distribution reflects the steric maximal possible conformational space of the labels that provides the outer limit. If the experimental value is not within the calculated distribution in one of the four plots the structure is ruled out.

Fig. 3 (A-D) represents the distance calculation for the seven published dimer models shown in Fig. S1. The bandwidths of the calculated curves reflect the outer limit of label flexibility for one single dimer. In contrast, the experimental bandwidth is the standard deviation of the average value of the protein ensembles ( 60 million proteins per measurement) of eight measurements for two different samples. Therefore, the experimental bandwidth is substantially narrower than the calculated one. However, only for dimer model I.1 and I.2 the calculated distance distributions overlap with all four experimental distances. Model I.3 does not match the T124-T124 measurements, while model I.4 does not match the ones for T124-T124 and nucleotide- 
nucleotide. Model II.1 is in accordance with the nucleotide distance measurements and significantly out of the experimental region for the other three distances, while model II.2 is only in accordance with the S106-S106 distance. Model III.1 diverges significantly from the nucleotidenucleotide distance. Besides models I.1 and I.2 only I.5 to 1.9 are in accordance with all four values as revealed by the comparison of the 64 dimer conformation obtained through protein docking in Table S3, while models I.10 to I.13 present only one value which deviates slightly from the four measurements. The rest of the obtained conformations displayed significantly different values compared to the experimental distances. Consistently, the category II and III models obtained for K-Ras4B do not match the distances for K-Ras4B-GTP (Table S4) or K-Ras4B-GDP (Table S5) that were derived from published NMR shifts.

Based on their agreement with the experimental data, we probed the stability and further refined the detailed dimer model interaction interface of the conformations of category I through MD simulations, with the exception of I.2, that was already obtained by refinement through MD simulations ${ }^{18}$, as well as $\mathrm{I} .3$ and I.4 that were exclusively obtained for K-Ras4B whereas our experiments use $\mathrm{N}$-Ras.

\section{MD simulations used to obtain one equilibrated representative dimer model}

In total nine MD simulations of N-Ras-GDP initiated by the dimer models of conformation I.1 and $\mathrm{I} .5$ to $\mathrm{I} .12$ were prepared, ran for at least $550 \mathrm{~ns}$. Simulations were setup, performed and analyzed as described in supporting note 6 . Contact patterns were identified using PyContact ${ }^{42}$ and the Maximoby contact matrix algorithm. Simulation times and system properties are summarized in Table S6. Equilibration of the systems was checked based on the root mean square displacement (RMSD) from the respective starting structure of each conformation and the time evolution of the contact pattern (Fig. S6). The distances between the ensembles of the labels attached to the equilibrated representative structures were calculated and compared to the experimental values (Table S7) analog to the procedure for the predicted docking structures. Simulations 1.9 and 1.10 converge to an almost identical representative equilibrium dimer structural model (Fig. 4), that is in total accordance with the four experimental distances mentioned above. All other seven simulations converge to equilibrium structures with almost identical monomer orientation among each other but. slightly different compared to the equilibrium structures observed for 1.9 and I.10 (Fig. S7). The monomers are slightly tilted against each other, leading to divergence from the experimental nucleotide-nucleotide distance.

The time course analysis of the dimer interface interaction pattern of simulation trajectories 1.9 and I.10 identified a stable central salt bridge between D154 and R161. This contact was the key dimer stabilizing salt bridge in five out of the seven simulations leading to the second equilibrium structure. The contact between E49 and $\mathrm{H} 131$ is the second most prevalent contact involved in dimer interface formation either through a hydrogen bond or van-der-Waals interactions. However, this contact is fluctuating over time due to its exposed position at the edge of the dimer contact interface resulting in competing solvent interactions. Therefore, this contact is less stable than the D154/R161 contact, which is deeply buried in the center of the dimer interface (Fig. S7). A detailed list of the relevant intra-protein contacts observed in the simulation trajectories is provided in Table S8. 
The time course analysis of the dimer interface interaction pattern of simulation trajectories 1.9 and I.10 identified a stable central salt bridge between D154 and R161. This contact was the key dimer stabilizing salt bridge in five out of the seven simulations leading to the second equilibrium structure. The contact between E49 and $\mathrm{H} 131$ is the second most prevalent contact involved in dimer interface formation either through a hydrogen bond or van-der-Waals interactions. However, this contact is fluctuating over time due to its exposed position at the edge of the dimer contact interface resulting in competing solvent interactions. Therefore, this contact is less stable than the D154/R161 contact, which is deeply buried in the center of the dimer interface (Fig. S7). A detailed list of the relevant intra-protein contacts observed in the simulation trajectories is provided in Table S8.

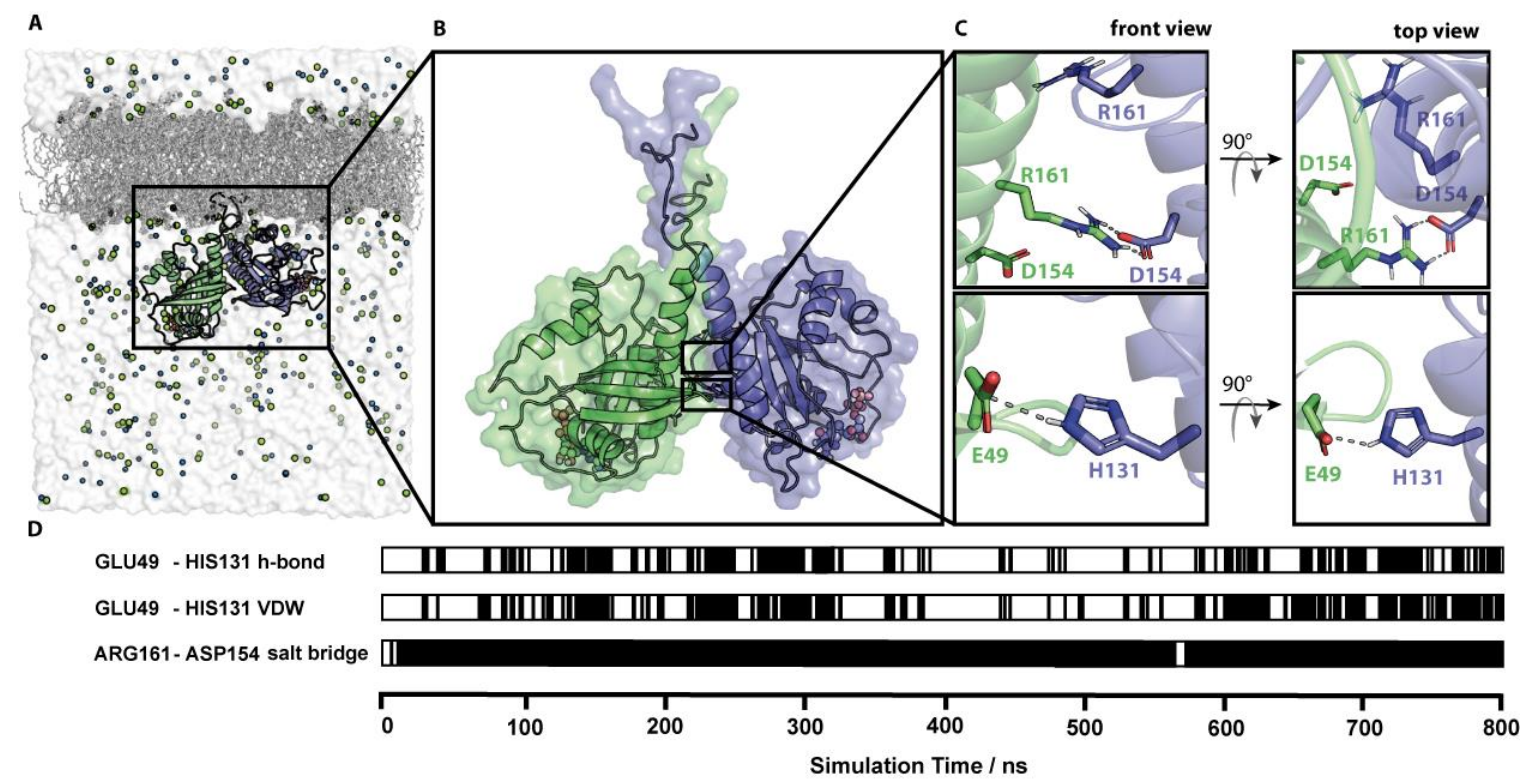

Fig. 4. Simulation of a membrane-bound N-Ras dimer. (A) The simulation system of a lipidated N-Ras dimer structural model attached to a POPC membrane inside a solvation box with physiological salt $(\mathrm{NaCl})$ concentration. (B) Representative protein structure of an 800 ns long MD simulation started with conformation 1.10 (representative structure in accordance with all four experimentally measured distances). (C) Key residues of the protein-protein interaction network identified by contact analysis of the simulation trajectory. (D) The time course of the key contacts within the simulation of conformation I.10. All contacts and the root mean square displacement (RMSD) are shown in Fig. S6.

Validation of the predicted dimer interface by FRET measurements of the E49Q D154N NRas variant

The MD simulations revealed that the most prominent dimer contact is the salt bridge between D154 and R161 and the second most prominent interaction is between E49 and H131. Based on our predicted contacts, we expected the $\mathrm{E} 49 \mathrm{Q}$ and $\mathrm{D} 154 \mathrm{~N}$ mutations in Ras to prevent dimerization (Fig. 5A). To validate our predicted contact interface, we performed FRET measurements of the lipidated membrane- bound N-Ras E49Q D154N variant. As the FRET signal with fluorophores at residue 106 was the most prominent in wild type, we chose this label position for our variant. No FRET signal was observed (Fig. 5B) for the N-Ras E49Q D154N 
variant, demonstrating that the E49Q D154N is the variant that prevents dimer formation. This dimer preventing variant provides a clear-cut experimental proof for our suggested dimer model.

A

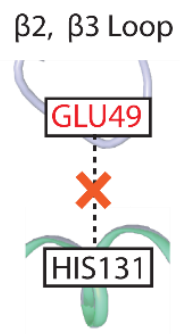

a-Helix 4

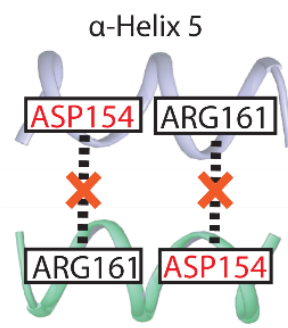

a-Helix 5

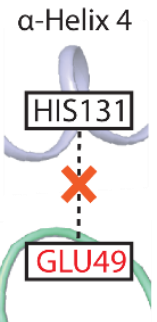

$\beta 2, \beta 3$ Loop

B S106-S106 Ras E49Q D154N C S106-S106 Ras wt
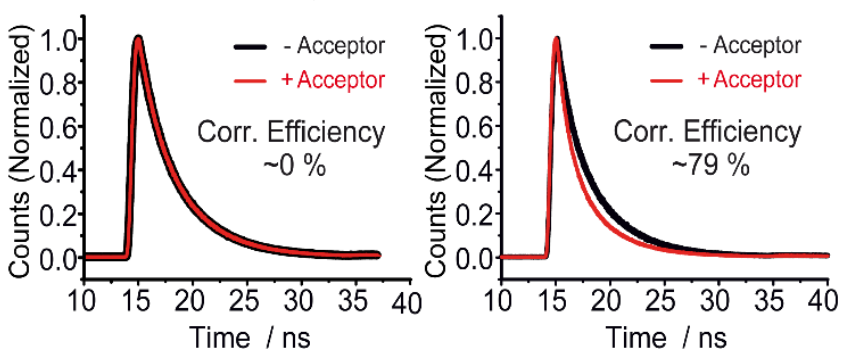

Fig. 5. Dimerization preventing E49Q D154N N-Ras variant. (A) Schematic summary of the key dimer interactions (Table S6) obtained from MD simulations (Table S7) suggesting E49 and D154 as promising candidates for dimer preventing mutations. (B) FRET measurements of lipidated membrane-bound N-Ras E49Q D154N variant confirm that mutations of E49 and D154 prevent dimer formation as no observable FRET signals were detected compared to N-Ras WT (C). The computationally predicted $\mathrm{N}$-Ras dimer interface is experimentally validated.

\section{Discussion}

The organization of Ras at the plasma membrane is decisive for its function. There is experimental evidence that Ras dimerization is crucial for nanoclustering at the plasma membrane and signal transmission ${ }^{35}$. Our experiments with lipidated N-Ras WT attached to a POPC membrane (Fig. 2) provide further evidence for dimerization due to the evoked FRETefficiencies or DEER signals (Table S2 and Fig. S3). Our in vitro experimental setup using a POPC membrane does not perfectly match the in vivo conditions; however we are convinced that our found dimer interface is transferable to in vivo conditions. Dimerization of membrane-bound Ras was also shown for other completely different artificial membrane systems such as nanodiscs by $\mathrm{NMR}^{32}$ Even more important, an in vivo study showed that an impaired dimerization of oncogenic K-Ras abolishes tumor growth ${ }^{22}$. Dimerization at a membrane is indicated by the majority of experimental studies as previously described. The two most relevant studies for this matter indicate that K-Ras-GDP and K-Ras-GTP form dimers at a physiological expression level as revealed by quantitative photoactivated localization microscopy. ${ }^{20,19}$ These studies observed a connection between the Ras dimerization and the activation of the Raf-MAPK signal pathway. Furthermore, attenuated total reflectance (ATR) infrared spectroscopy measurements in 
combination with biomolecular simulations have shown that $\mathrm{N}$-Ras dimerizes on POPC membranes. ${ }^{18}$ Some studies provide conflicting results that exclude dimerization, most of which, were performed without a membrane in their experimental setup. ${ }^{25}$ The only biophysical study indicating monomeric Ras, while including a membrane, measured the diffusion of Ras in a solid supported bilayer in vitro. ${ }^{26}$ The authors of the study stated that Ras dimerization in vivo must depend on more factors than the presence of the protein alone. Furthermore, they noted that the glass support of the bilayer may damp large membrane undulations influencing the lateral organization of Ras leading to contradicting results compared to the aforementioned in vivo studies.

There have been valid concerns that dimerization shown by FRET measurements is associated with high laser intensities ${ }^{26}$ or high protein concentrations. However, our measurements show that neither of it is the case for the used experimental setup. While high laser intensities are exerted by confocal microscopes, we used time correlated single photon counting (TCSPC) with low laser intensity or EPR which does not involve a laser. The concern regarding protein concentration, was eliminated since we obtained dimerization for one measurement (N-Ras WT S106-S106) and no dimerization for another (E49Q D154N N-Ras variant S106-S106) while using the same protein concentration and identical experimental setups. Thus, there is strong evidence that lipidated membrane-bound full-length Ras forms dimers.

The lack of a detectable FRET signal for the E49Q D154N N-Ras variant indicates that this interaction site is the only one present, as at least a somewhat visible FRET signal would then be expected. This implies that full-length $\mathrm{N}$-Ras does not form oligomers at POPC membranes, as an additional interaction site, different from the dimer interaction site identified here, would be necessary to form oligomers in wt N-Ras.

Our results indicate a decisive role of the lipid anchor in dimerization with and without membrane. We obtained comparable FRET efficiencies of full-length lipidated N-Ras solely in solution (S106S106: $60 \%$ and T124-T124: 10\%) and membrane bound (S106-S106: 79\% and T124-T124: 15\%). However, the same FRET measurements in solution using N-Ras 1-181 without the lipid anchor showed no signal, indicating that the Ras $G$ domain lacks the intrinsic propensity to form dimers in solution. The N-Ras anchor is very hydrophobic (Fig. S8), indicating that the hydrophobic effects are driving forces for anchor dimerization. These findings are in line with previous studies on the self-association of the $G$ domain in solution observed via analysis of the time-domain fluorescence anisotropy and NMR chemical shift perturbations. ${ }^{30}$ Overall, our data clearly indicate that the N-Ras lipid anchor is essential for dimer formation in solution. We hypothesize that clustering of the lipid anchor is the first step towards dimerization. Clustering of the lipid anchor brings the $\mathrm{G}$ domains closer together and thus increases the local concentration of Ras proteins, which leads to the formation of one single otherwise comparably low affinity $G$ domain interface.

Gasper and Wittinghofer suggested as an argument against dimerization that different Ras crystals show different crystal packings. ${ }^{3}$ The $\mathrm{C}$-terminal hypervariable region (HVR) including the lipid anchor is truncated in crystal structures due to their high flexibility. The remaining $G$ domain has the potential to sample and form a huge variety of different dimer interfaces, due to the high protein concentrations during crystallization. This variety of dimer structural models is in line with the high variety of dimer conformations found by protein-protein docking algorithms (Table S1). Both crystallography and docking studies miss the native conformational space restrictions as clustering of the lipid anchor and the space occupied by the membrane itself allowing only limited possible G-domain dimer conformations for full length Ras. Therefore, crystal structures and protein-protein docking results lack the capacity to provide a single prominent structure, which we 
observe here for full length N-Ras. We note that our proposed dimer model is also a prominent motive within the crystal packing of Ras crystal structures. Thus, the lipid anchor steers the protein into an exclusive dimer structure at the membrane.

A

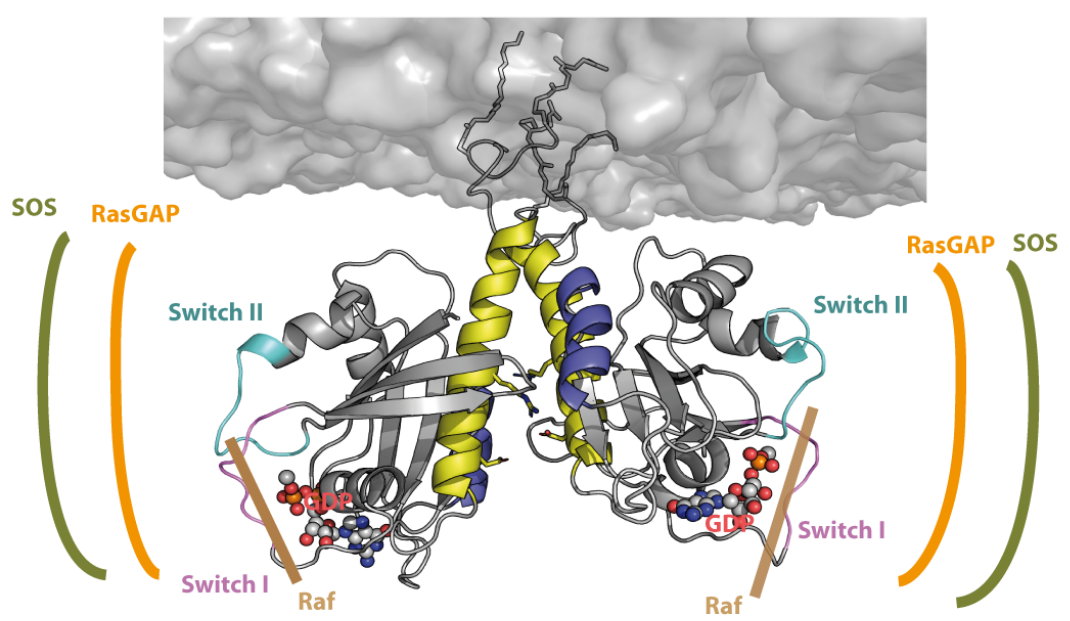

B

Switch I

Switch II

\begin{abstract}
K-RaS4A MTEYKLVVVGAGGVGKSALTIQLIQNHFVDEYDPTIEDSYRKQVVIDGETCLLDILDT K-Ras4B MTEYKLVVVGAGGVGKSALTIQLIQNHFVDEYDPTIEDSYRKQVVIDGETCLLDILDTAGQEEY 64 N-Ras MTEYKLVVVGAGGVGKSALTIQLIQNHFVDE YDPTIEDSYRKQVVIDGE TCLIDILDTAGQEEY 64 H-Ras MTEYKLVVVGAGGVGKSALTIQLIQNHFVDEYDPTIEDSYRKQVVIDGE TCLIDILDTAGQEEY 64
\end{abstract}

K-RaS4A SAMRDOYMRTGEGFLCVFAINNTKSFEDIHHYREOIKRVKDSEDVPMVLVGNKCDLPSRTVDTK 128 K-Ras4B SAMRDQYMRTGEGFLCVFAINNTKSFEDIHHYREQIKRVKDSEDVPMVLVGNKCDLPSRTVDTK 128 $\begin{array}{ll}\text { K-Ras4B } & \text { SAMRDQYMRTGEGFLCVFAINNTKSFEDIHHYREQIKRVKDSEDVPMVLVGNKCDLPSRTVDTK } 128 \\ \text { N-Ras } & \text { SAMRDQYMRTGEGFLCVFAINNSKSFADINLYREQIKRVKDSDDVPMVLVGNKCDLPTRTVDTK } 128\end{array}$ H-Ras SAMRDQYMRTGEGFLCVFAINNTKSFEDIHQYREQIKRVKDSDDVPMVLVGNKCDLAARTVESR 128

K-Ras4A QAODLARSYGIPFIETSAKTRORVEDAFYTLVRE IROYRLKKISKE-EKTPGCVKIKKCIIM 189 K-Ras4B OAODLARSYGIPFIETSAKTROGVDDAFYTLVRE IRKHKEKM-SKD-GKKKKKKSKTKCVIM 188 N-Ras QAHELAKSYGIPFIETSAKTRQGVEDAFYTLVRE IRQYRMKKLNSSDDGTQGCMGLP-CVVM 189 H-Ras QAQDLARSYGIPYIETSAKTRQGVEDAFYTLVRE IRQHKLRKLNPPDE SGPGCMSCK-CVLS 189 D154 R161

Fig. 6. N-Ras dimer structural model and its interaction sites. (A) N-Ras dimer structural model in accordance with all four experimentally observed distances. The dimer interface is formed by helices a4 (blue) and a5 (yellow). The binding sites of SOS (green), Ras-GAP (orange) and Raf (brown) are accessible for the respective Ras interaction partners. The nucleotide binding site and the switch I (pink) and II (cyan) are not involved into the dimer interface as they are on the opposite side of the dimer interface. (B) Alignment of all Ras isoforms with nonconserved amino acids highlighted in light gray. The key contact residues D154/R161 (green) stabilizing the Ras dimer (Fig 4 and 5) are conserved among all Ras isoforms.

Knowledge of the precise amino acid interaction network of the dimer interface provides novel target sites for anti-cancer drugs that prevent Ras dimerization, which plays a crucial role in signal transduction in vivo. ${ }^{36}$ Our study shows for $\mathrm{N}$-Ras that only those dimer models exhibiting an interface involving helices a4 and a5 (dimer category I) are in accordance with all four experimental distances. Dimer category II disagrees with both FRET and EPR distance obtained for position T124 and category III disagrees with the experimentally observed nucleotidenucleotide distance. In addition, Fig. 6 shows that the binding sites for the three Ras interaction partners Raf, RasGAP and SOS are all accessible for dimer category I structural models. NMR 
distances obtained for K-Ras also show that helices a 4 a5 are involved in dimerization. ${ }^{32}$ The isoform independent motive of helices $\alpha 4$ and $\alpha 5$ dimerization interface is supported by a study showing the impact of disrupting this interface with a small synthetic protein affecting the signaling of both H-Ras and K-Ras. ${ }^{19}$ The importance of dimerization for in vivo signaling is emphasized by the study showing that the disruption of the oncogenic K-RasG12D dimer abolishes in vivo tumor growth. ${ }^{22}$ Taking together our results and the previously mentioned reports, we propose that the formation of the dimer interface by helices $\alpha 4$ and $\alpha 5$ is a universally valid motive for all Ras isoforms.

Biomolecular simulations revealed one representative equilibrated lipidated membrane-bound full-length N-Ras-GDP dimer model (Fig. 6) that is in accordance with all experimental distances (Table S7). The identified key residues (Fig. 4) forming the salt bridge D154/R161, are conserved among all Ras isoforms. This salt bridge was also identified as a key contact in our previously published simulations on $\mathrm{N}$-Ras. ${ }^{18}$ The second most prominent contact residue E49 is also conserved and its interaction partner $\mathrm{H} 131$ is exchanged to Q131 in $\mathrm{H}$ - and K-Ras, still retaining the ability to form a hydrogen bond to E49. All these contact residues are positioned within a flat area of the Ras surface (Fig. S7), which supports the proposed dimer interface. For N-Ras we confirmed the importance of D154 and E49 for dimerization as the E49Q D154N mutations prevented dimerization (Fig. 5). Some but not all of the observed K-Ras4B NMR shifts ${ }^{32}$ are in agreement with our N-Ras models refined through biomolecular simulations (Table S4 and S5). These deviations indicate a difference between N-Ras and K-Ras4B. Furthermore, model I.3 and I.4 for K-Ras4B do not exhibit the D154/R161 key contact. Further experimental proof regarding the presence of the D154/R161 interaction in K-Ras4B is provided by Ambrogio et al. ${ }^{22}$ They show the importance of the D154/R161 contact in K-Ras4B. Each of the two single mutants D154Q and R161E of oncogenic K-Ras4BG12D abolished in vivo tumor growth, Implying that this is due to the impaired ability to form dimers. Thus, mutations in the dimer interface have the potential to eliminate the effect of oncogenic mutants at positions G12, G13, or Q61. The contact swapping mutant D154Q R161E showed tumor growth, though not as high as the wild type, possibly due to a weaker contact between Gln and Glu as opposed to the contact between Glu and Arg, though still allowing for dimer formation. There might be slight difference in the detailed dimer interaction pattern between the Ras isoforms that still need further investigation. As shown by Spencer-Smith and colleagues, charge reversal mutation of D154 or R161 and R135 exhibited no effect on oncogenic H-RasG12V signaling, indicating the stabilization of its dimer interface through other residues for this isoform. ${ }^{19}$ We anticipate that $\mathrm{N}$-Ras and K-Ras both dimerize through helices $\alpha 4$ and $\alpha 5$ with D154/R161 as the key contact; however, further research is required to unravel the detailed $\mathrm{H}$-Ras dimer interaction pattern.

In summary, we developed a novel strategy to incorporate unnatural amino acids into Ras, allowing for two orthogonal site-specific modifications of the same protein (Fig 2); one to attach a lipid anchor and a second one for fluorophores or spin labels for distance measurements. Exploiting the gained flexibility of individual site-specific labeling we obtained three novel experimental distance information about N-Ras dimers. The S106-S106 distance of $43.3 \pm 2.3 \AA$ and T124-T124 distance of $72.0 \pm 3.5 \AA$ through FRET measurements and another T124-T124 distance of with $59.6 \pm 4.4 \AA$ by EPR spectroscopy. All measurements demonstrate conclusively that lipidated full length $\mathrm{N}$-Ras dimerizes at POPC membranes. We show that among 178 structural dimer models predicted by different protein-protein docking algorithms only those including helices $\alpha 4$ and $\alpha 5$ in the dimer interface are in accordance with the here obtained FRET 
and EPR data as well as previous FRET distances. ${ }^{18}$ Further refinement of the dimer interface through biomolecular simulations revealed that the salt bridge between D154/R161 together with the interactions of E49 with $\mathrm{H} 131$ are the key $\mathrm{N}$-Ras dimerization contacts (Fig. 4). Our computationally predicted interface was experimentally validated as FRET measurements showed that the N-Ras E49Q D154N variant prevented dimerization (Fig. 5).

Taking together our results for N-Ras, the sequence similarities between the isoforms, and the described literature for $\mathrm{H}$-Ras and $\mathrm{K}$-Ras we propose that all Ras isoforms dimerize at the membrane through helices $a 4$ and a5 (Fig. 6). Nevertheless, there might be isoform specific differences in the exact orientation of the monomers. These atomic resolution structural insights into the Ras dimer interface provide novel target sites to develop anti-cancer drugs inhibiting Ras signaling by preventing its dimerization.

\section{Materials and Methods}

\section{Experimental Methods}

Biochemical details of lipid anchor synthesis, sample preparation of lipidated N-Ras with incorporated unnatural amino acids, coupling of fluorophores, and sample composition for FRET and EPR measurements are described in supporting note 3.

The TCSPC histograms were evaluated, and the FRET efficiencies were calculated as described in supporting note 4 . The results are also displayed in Table S2. In the presence of the fluorescence acceptor, the amplitude weighted and averaged fluorophore's lifetimes decreased from 1.87 ns to $1.28 \mathrm{~ns}$ in measurements with membrane-bound N-Ras (GDP) S106-Atto532 and from 2.66 ns to $2.53 \mathrm{~ns}$ in measurements with membrane-bound N-Ras (GDP) T124-Atto532. As detailed in Supporting Note 4, we have to consider that dimers are randomly formed between donor labeled Ras (D), acceptor labeled Ras (A) and unlabeled Ras. Presuming a dimer and considering the fraction of DA dimers these values lead to FRET efficiencies of $~ 79 \%$ (S106S106) and $15 \%$ (T124-T124).

We have performed the same experiment using proteins without lipid anchor, but in presence of POPC liposomes, and did not observe any FRET signals. This shows that the G-domain without the anchor does not form stable dimers. Measurements on lipidated protein without POPC liposomes resulted in slightly lower FRET efficiencies as described above. Here, the amplitude weighted and averaged fluorophore's lifetime decreased from $1.86 \mathrm{~ns}$ to $1.41 \mathrm{~ns}$ in measurements with Ras S106-Atto532 and from 3.56 ns to 3.44 ns in measurements with Ras T124-Atto532 leading to FRET efficiencies of $\sim 60 \%$ for S106-S106 and $~ 10 \%$ for T124-T124. This suggests, that lipidated Ras proteins in solution interact with each other via the lipid anchor.

Experimental details of the EPR measurements are given in supporting note 7.

\section{Computational Methods}

The protein-protein docking approach is described in supporting note 2. The protocol to calculate distance distributions for the predicted models employing the PyMOL plugin for MTSSL Wizard ${ }^{43}$ is described in supporting note 5 .

Biomolecular simulations were prepared using MAXIMOBY (CHEOPS, Germany) and ran with Gromacs $2019^{44}$. The structure preparation and the used protocol including all parameters for 
simulations runs are described in supporting note 6 . Simulation results were visualized using VMD 1.9.4 $4^{45}$ and PyMOL 2.2. Simulation trajectories were analyzed using the analysis tools of Gromacs 201944, VMD 1.9.4, and QwikMD ${ }^{46}$. Contact patterns were identified using PyContact ${ }^{42}$ and the MAXIMOBY (CHEOPS, Germany) contact matrix algorithm. Details about the input structures and the analysis of the biomolecular simulations are provided in supporting note 6 and supporting table S6.

\section{Acknowledgments}

We thank Herbert Waldmann and Sascha Gentz for their support with synthesizing the lipid anchor. We thank Iris Bourdos for her support in the creation of N-Ras mutants and expression tests. We thank Léon Beyer and Konstantin Gavriljuk for MALDI measurements and Gregor Hagelueken for technical support with the MTSSL Wizard. The plasmids encoding tRNA ${ }^{\text {Pyl/PyIRS }}{ }^{\text {WT/AF }}$ were obtained through an MTA with Prof. Dr. Edward Lemke (Johannes Gutenberg University, Biocentre \& IMB, Mainz).

\section{Funding}

This work was supported by Deutsche Forschungsgemeinschaft (DFG, German Research Foundation) Individual Research Grant GE 599/20-1 to KG and KO 3813/1-1 to CK. Further support was provided by the Ministry for Culture and Science (MKW) of North Rhine-Westphalia (Germany) through grant 111.08.03.05-133974 to KG and the Protein Research Unit Ruhr within Europe (PURE) funded by the Ministry of Innovation, Science and Research (MIWF) of NorthRhine Westphalia (Germany) to KG.

\section{Author Contributions}

TR, KG, and CK designed the research; TR, CT, MS, and JK performed research; CT performed FRET measurements and labeling experiments supervised by CK. TR and MS performed MD simulations. MS performed protein docking, and MTSSL Wizard calculations supervised by TR. JG assisted with the MTSSL Wizard analysis. JS synthesized the lipid anchor. JK performed EPR experiments and related MD simulations. ML assisted with protein expression. TR, CT, MS, JG, JK, and CK analyzed data; TR, CT, MS, KG, and CK wrote the paper with edits from all co-authors. KG and CK obtained funding.

\section{References}

1. Mishra AK, Lambright DG. Invited review: Small GTPases and their GAPs. Biopolymers 2016; 105(8):431-48.

2. Scheffzek K, Ahmadian MR, Kabsch W, Wiesmüller L, Lautwein A, Schmitz F et al. The RasRasGAP complex: structural basis for GTPase activation and its loss in oncogenic Ras mutants. Science (New York, N.Y.) 1997; 277(5324):333-8.

3. Gasper R, Wittinghofer F. The Ras switch in structural and historical perspective. Biological chemistry 2019; 401(1):143-63.

4. Thapar R, Williams JG, Campbell SL. NMR characterization of full-length farnesylated and nonfarnesylated H-Ras and its implications for Raf activation. Journal of molecular biology 2004; 343(5):1391-408.

5. Kötting C, Güldenhaupt J, Gerwert K. Time-resolved FTIR spectroscopy for monitoring protein dynamics exemplified by functional studies of Ras protein bound to a lipid bilayer. Chemical 
Physics 2012; 396:72-83. Available from: URL:

http://www.sciencedirect.com/science/article/pii/S0301010411003636.

6. Calixto AR, Moreira C, Pabis A, Kötting C, Gerwert K, Rudack T et al. GTP Hydrolysis Without an Active Site Base: A Unifying Mechanism for Ras and Related GTPases. Journal of the American Chemical Society 2019; 141(27):10684-701.

7. Cox AD, Der CJ. Ras history: The saga continues. Small GTPases 2010; 1(1):2-27.

8. Lu S, Jang H, Muratcioglu S, Gursoy A, Keskin O, Nussinov R et al. Ras Conformational Ensembles, Allostery, and Signaling. Chemical reviews 2016; 116(11):6607-65.

9. Prior IA, Hancock JF. Ras trafficking, localization and compartmentalized signalling. Seminars in cell \& developmental biology 2012; 23(2):145-53.

10. Simanshu DK, Nissley DV, McCormick F. RAS Proteins and Their Regulators in Human Disease. Cell 2017; 170(1):17-33. Available from: URL:

https://pubmed.ncbi.nlm.nih.gov/28666118/.

11. Maegley KA, Admiraal SJ, Herschlag D. Ras-catalyzed hydrolysis of GTP: a new perspective from model studies. Proceedings of the National Academy of Sciences of the United States of America 1996; 93(16):8160-6.

12. Ahmadian MR, Stege $P$, Scheffzek K, Wittinghofer A. Confirmation of the arginine-finger hypothesis for the GAP-stimulated GTP-hydrolysis reaction of Ras. Nat Struct Mol Biol 1997; 4(9):686-9.

13. Gerwert K, Mann D, Kötting C. Common mechanisms of catalysis in small and heterotrimeric GTPases and their respective GAPs. Biological chemistry 2017; 398(5-6):523-33.

14. Moore AR, Rosenberg SC, McCormick F, Malek S. RAS-targeted therapies: is the undruggable drugged? Nature reviews. Drug discovery 2020; 19(8):533-52. Available from: URL:

https://pubmed.ncbi.nlm.nih.gov/32528145/.

15. Canon J, Rex K, Saiki AY, Mohr C, Cooke K, Bagal D et al. The clinical KRAS(G12C) inhibitor AMG 510 drives anti-tumour immunity. Nature 2019; 575(7781):217-23.

16. Goebel L, Müller MP, Goody RS, Rauh D. KRasG12C inhibitors in clinical trials: a short historical perspective [RSC Medicinal Chemistry, 11(7), 760-770]. RSC Med. Chem. 2020; 11(7):760-70.

17. Inouye K, Mizutani S, Koide H, Kaziro Y. Formation of the Ras dimer is essential for Raf-1 activation. The Journal of biological chemistry 2000; 275(6):3737-40.

18. Güldenhaupt J, Rudack T, Bachler P, Mann D, Triola G, Waldmann H et al. N-Ras forms dimers at POPC membranes. Biophys J 2012; 103(7):1585-93.

19. Spencer-Smith R, Koide A, Zhou Y, Eguchi RR, Sha F, Gajwani P et al. Inhibition of RAS function through targeting an allosteric regulatory site. Nature chemical biology 2017; 13(1):628.

20. Nan X, Tamgüney TM, Collisson EA, Lin L-J, Pitt C, Galeas J et al. Ras-GTP dimers activate the Mitogen-Activated Protein Kinase (MAPK) pathway. Proceedings of the National Academy of Sciences of the United States of America 2015; 112(26):7996-8001. 
21. Chen M, Peters A, Huang T, Nan X. Ras Dimer Formation as a New Signaling Mechanism and Potential Cancer Therapeutic Target. Mini reviews in medicinal chemistry 2016; 16(5):391-403.

22. Ambrogio C, Köhler J, Zhou Z-W, Wang H, Paranal R, Li J et al. KRAS Dimerization Impacts MEK Inhibitor Sensitivity and Oncogenic Activity of Mutant KRAS. Cell 2018; 172(4):857-868.e15.

23. Muratcioglu S, Chavan TS, Freed BC, Jang H, Khavrutskii L, Freed RN et al. GTP-Dependent KRas Dimerization. Structure (London, England : 1993) 2015; 23(7):1325-35.

24. Rhett JM, Khan I, O'Bryan JP. Biology, pathology, and therapeutic targeting of RAS. Advances in cancer research 2020; 148:69-146.

25. Kovrigina EA, Galiakhmetov AR, Kovrigin EL. The Ras G Domain Lacks the Intrinsic Propensity to Form Dimers. Biophys J 2015; 109(5):1000-8. Available from: URL:

https://pubmed.ncbi.nlm.nih.gov/26331257/.

26. Chung JK, Lee YK, Denson J-P, Gillette WK, Alvarez S, Stephen AG et al. K-Ras4B Remains Monomeric on Membranes over a Wide Range of Surface Densities and Lipid Compositions. Biophys J 2018; 114(1):137-45.

27. Chung JK, Lee YK, Lam HYM, Groves JT. Covalent Ras Dimerization on Membrane Surfaces through Photosensitized Oxidation. Journal of the American Chemical Society 2016; 138(6):1800-3.

28. Plowman SJ, Muncke C, Parton RG, Hancock JF. H-ras, K-ras, and inner plasma membrane raft proteins operate in nanoclusters with differential dependence on the actin cytoskeleton. Proceedings of the National Academy of Sciences of the United States of America 2005; 102(43):15500-5.

29. Prior IA, Muncke C, Parton RG, Hancock JF. Direct visualization of Ras proteins in spatially distinct cell surface microdomains. The Journal of cell biology 2003; 160(2):165-70.

30. Barklis E, Stephen AG, Staubus AO, Barklis RL, Alfadhli A. Organization of Farnesylated, Carboxymethylated KRAS4B on Membranes. Journal of molecular biology 2019; 431(19):370617.

31. Prakash P, Sayyed-Ahmad A, Cho K-J, Dolino DM, Chen W, Li H et al. Computational and biochemical characterization of two partially overlapping interfaces and multiple weak-affinity K-Ras dimers. Sci Rep 2017; 7(1):40109. Available from: URL:

https://www.nature.com/articles/srep40109.

32. Lee K-Y, Fang Z, Enomoto M, Gasmi-Seabrook G, Le Zheng, Koide S et al. Two Distinct Structures of Membrane-Associated Homodimers of GTP- and GDP-Bound KRAS4B Revealed by Paramagnetic Relaxation Enhancement. Angewandte Chemie (International ed. in English) 2020; 59(27):11037-45.

33. Tian T, Harding A, Inder K, Plowman S, Parton RG, Hancock JF. Plasma membrane nanoswitches generate high-fidelity Ras signal transduction. Nature cell biology 2007; 9(8):90514. Available from: URL: https://pubmed.ncbi.nlm.nih.gov/17618274/.

34. Tian T, Plowman SJ, Parton RG, Kloog Y, Hancock JF. Mathematical modeling of K-Ras nanocluster formation on the plasma membrane. Biophys J 2010; 99(2):534-43. 
35. Zhou Y, Hancock JF. Ras nanoclusters: Versatile lipid-based signaling platforms. Biochimica et biophysica acta 2015; 1853(4):841-9.

36. Zuberi M, Khan I, O'Bryan JP. Inhibition of RAS: proven and potential vulnerabilities.

Biochemical Society transactions 2020.

37. Baspinar A, Cukuroglu E, Nussinov R, Keskin O, Gursoy A. PRISM: a web server and repository for prediction of protein-protein interactions and modeling their 3D complexes. Nucleic acids research 2014; 42(Web Server issue):W285-9.

38. Pierce BG, Wiehe $\mathrm{K}$, Hwang H, Kim B-H, Vreven T, Weng Z. ZDOCK server: interactive docking prediction of protein-protein complexes and symmetric multimers. Bioinformatics (Oxford, England) 2014; 30(12):1771-3.

39. Schneidman-Duhovny D, Inbar Y, Nussinov R, Wolfson HJ. PatchDock and SymmDock: servers for rigid and symmetric docking. Nucleic acids research 2005; 33(Web Server issue):W363-7.

40. Schneidman-Duhovny D, Inbar Y, Nussinov R, Wolfson HJ. Geometry-based flexible and symmetric protein docking. Proteins 2005; 60(2):224-31.

41. Plass T, Milles S, Koehler C, Schultz C, Lemke EA. Genetically encoded copper-free click chemistry. Angewandte Chemie (International ed. in English) 2011; 50(17):3878-81.

42. Scheurer M, Rodenkirch P, Siggel M, Bernardi RC, Schulten K, Tajkhorshid E et al. PyContact: Rapid, Customizable, and Visual Analysis of Noncovalent Interactions in MD Simulations. Biophys J 2018; 114(3):577-83.

43. Hagelueken G, Ward R, Naismith JH, Schiemann O. Mtss IWizard: In Silico Spin-Labeling and Generation of Distance Distributions in PyMOL. Applied magnetic resonance 2012; 42(3):37791.

44. Abraham MJ, Murtola T, Schulz R, Páll S, Smith JC, Hess B et al. GROMACS: High performance molecular simulations through multi-level parallelism from laptops to supercomputers. SoftwareX 2015; 1-2:19-25. Available from: URL: http://www.sciencedirect.com/science/article/pii/S2352711015000059.

45. Humphrey W, Dalke A, Schulten K. VMD: Visual molecular dynamics [Journal of Molecular Graphics, 14(1), 33-38]. Journal of Molecular Graphics 1996; 14(1):33-8.

46. Ribeiro JV, Bernardi RC, Rudack T, Stone JE, Phillips JC, Freddolino PL et al. QwikMD Integrative Molecular Dynamics Toolkit for Novices and Experts. Sci Rep 2016; 6(1):26536. Available from: URL: https://www.nature.com/articles/srep26536. 